\title{
A stroboscopic view of Professor E. C. G. Sudarshan
}

\author{
K. R. Sreenivasan* \\ School of Mathematics, Institute for Advanced Study, Princeton, New Jersey 08540, USA
}

\section{Preamble}

Professor George Sudarshan's world-view was expansive, contemplative, deep and free-spirited - though, perhaps surprisingly, also combative and rocky on occasion. At the peak of his powers, he was an extraordinarily gifted theoretical physicist of his time. How to capture him in these few pages? It is especially important to ask this question because we both had met or interacted only around ten times in all. Since the occasions were sporadic, our conversations each time began at the beginning and did not build on previous ones. I was not his student or a scientific collaborator, and his research hardly overlapped with mine.

So I wrestled with this question considerably before agreeing to write, and came to these conclusions. I greatly admire George's deep science and guileless humanity. Though some of his traits have baffled me over time, I could easily have been a sympathetic colleague of his. Our conversations were infrequent, it is true, but his impact on my thought-world was disproportionately pervasive. More to the point here, when I discussed the possibility of this article with a few of George's friends, and took the time to re-read some of his work and much of his correspondence, my views of him did not seem unreasonable. I felt that I could say what little I will about his science and circumstances surrounding it, as well as his beliefs and actions - with a modicum of genuineness.

George's life, work and papers justify a full-fledged biography by someone truly knowledgeable. Mine is a selective reflection on his thoughts and essence, as I see them, and it is fair to sound the warning that this tribute is highly personal; but most things I have said of him below are a distillation of George's own words. He had his imperfections. But a person's worth is not just the sum of his or her positives and negatives; we should take note of the negatives for reasons of historical accounting, but sum up the positives as the legacy. Among the positives, 'excellence in favour of the median good' is a thought that George would have appreciated. I am not sure how to apologize for it.

\footnotetext{
*e-mail: katepalli.sreenivasan@nyu.edu
}

\section{My encounters with George Sudarshan}

A basic sense of integrity forces me to place on record the meagreness of our overlap. It is, of course, impossible not to be aware of George in one way or another, if one is part of the US physics community of Indian origin.

I was still a graduate student at the Indian Institute of Science (IISc), Bengaluru, when George made his remarkable entrance there as the Director of the Center for Theoretical Studies (CTS). He would visit CTS during summers, and sometimes in between.

I attended a few of George's lectures soon after, one of which may have been part of the conference on 'Unsolved Problems in Physics'. He was electrifying as a speaker. One of his other talks was something like, 'Why society should support theoretical physics', but it had nothing to do with sociology or policy-making or practical benefits of science to mankind, as one might have thought. It was about theoretical physics from the first sentence uttered, displaying erudition, depth and breadth, delivered with humour and candid irreverence towards such hallowed scientists as Einstein, Heisenberg, and Schrödinger. And, as an afterthought, he concluded with a well-known shloka ${ }^{1}$ followed by a mysterious statement, 'this is why society should support theoretical physics'. I had grown up in a tradition of paying homage to all past great scientists and to present one's work with overt seriousness, and had not witnessed such delightfully saucy lectures, so it swept away some of my cobwebs.

For reasons I cannot explain, I found myself in the same room as George on a few occasions in the next two or so years, in one of which Satish Dhawan, then the Director of IISc, gave a pep talk to IISc professors in attendance about doing quality science instead of focusing on quantity. He pointed to George and said, 'Here is a man who flies faster than light'. That was my introduction to tachyons, in 1972 or 1973 . I was introduced to his $\mathrm{V}-\mathrm{A}$ theory also in a strange way - but more of that later.

During his visits, George would sometimes sit on the steps of CTS (the former gymkhana building used just prior to CTS as medical dispensary), busy with thoughts and calculations; I would encounter him in this state while traversing from the hostel to my department. Once I dared to introduce myself, stating that I was Roddam Narasimha's student. He and Narasimha were on 
excellent terms and George knew that the latter was a student of Dhawan before his Caltech sojourn. George genuinely liked Dhawan, so I remember him saying that I had 'good pedigree'.

I left the country in 1975 and would hear of George mostly during my periodic visits to IISc. During one such visit, George and I happened to stay together at the main guesthouse, along with Raja Rao, his colleague at the University of Texas at Austin (UT Austin, USA), and a celebrated Indian author in English ${ }^{2}$. Their relationship was deep for some years. At the breakfast, they discoursed on topics such as consciousness, turiya-state, etc. As a dinner companion once, mostly a silent one, I found Raja Rao's responses to George's probing questions to be less deep than what George may have sought.

George received the 1998 Distinguished Scientist Award and the 2008 Life Time Achievement Award ${ }^{3}$ of the American Chapter of the Indian Physics Association. We were seated next to each other during the 1998 award. He was mellow on the occasion, repeated the pedigree remark verbatim, and inquired briefly about my work (the only time he evinced an interest). I remember spending more time discussing the people we knew in common, less about science.

I was dazzled when I read George's book with Tony Rothman, Doubt and Certainty ${ }^{4}$, and used the excuse to reacquaint myself with George, who was kind to respond promptly. One time at UT Austin, he mentioned with some pride the dinner that the University President had organized for him at the University Tower, an honour that had never been bestowed on any faculty member before. This was part of his 75 th birthday meeting in 2006 (ref. 5). The Tower is lit in red only when the UT Austin football team wins a home game, and it was lit red for George's special event. I knew much more about him by then, and was touched that this unique honour had pleased him.

A bit later when I happened to be in charge of the Dirac Medal at ICTP, I felt that George should have been a recipient years earlier. I talked about this with his longtime friend and colleague in UT Austin, Swadesh Mahajan, and together we organized the nomination. Luis Boya (Zaragoza), G. C. Hegerfeldt (Göttingen), Jeff Kimble (Caltech), John Klauder (Florida), R. Narasimha (Bangalore), T. V. Ramakrishnan (Bangalore), and Steven Weinberg (Austin) generously supported the effort ${ }^{6}$. The Medal finally did come through in 2009 (see Figure 1). It was an occasion for me to reacquaint myself with George and his work.

Finally, G. Bhamathi, George's widow and former professor of physics at Madras University, kindly permitted me to read his correspondence; her good-natured and unconditional cooperation allowed me to get a better glimpse of events behind the scene, from George's graduate student days to just before his death. Some of this material I have shared in this essay, in some fashion.

\section{Two pieces of George Sudarshan's science}

By the time I became aware of George, he had made his mark as a brilliant theoretical physicist with major accomplishments behind him, starting with his $\mathrm{Ph} \mathrm{D}$ thesis, whose epilogue I have reproduced as Figure 2. (It is clear that he was bending backwards in stating his claims.) I will describe two elements of his work briefly just enough to provide the context for his struggles of priority. Towards the very end, I will make a tangential remark on his quantum optics work.

\section{Particles beyond the light barrier: tachyons}

There was a young lady named Bright, Whose speed was far faster than light.

She went out one day,

In a relative way,

And returned the previous night!

Reginald Buller (ca. 1930)

Dhawan's reference to George's work on tachyons, mentioned earlier, propelled me to read, sometime soon after, the beautiful article by Bilaniuk et al. $^{7}$; I later worked through another paper by Bilaniuk and Sudarshan ${ }^{8}$.

Travelling faster than light has been a popular notion for many years (as the ditty above shows), and the first hypothesis regarding faster-than-light particles is attributed to Arnold Sommerfeld in 1904. But it was George and his colleagues who transformed the tantalizing notion into science. They were the ones who argued that tachyons have negative energy in certain inertial frames, and that a negative-energy tachyon travelling backward in time could be reinterpreted as a positive-energy particle travelling forward in time. Tachyons have imaginary rest mass and speed up as they lose their energy, and attain infinite

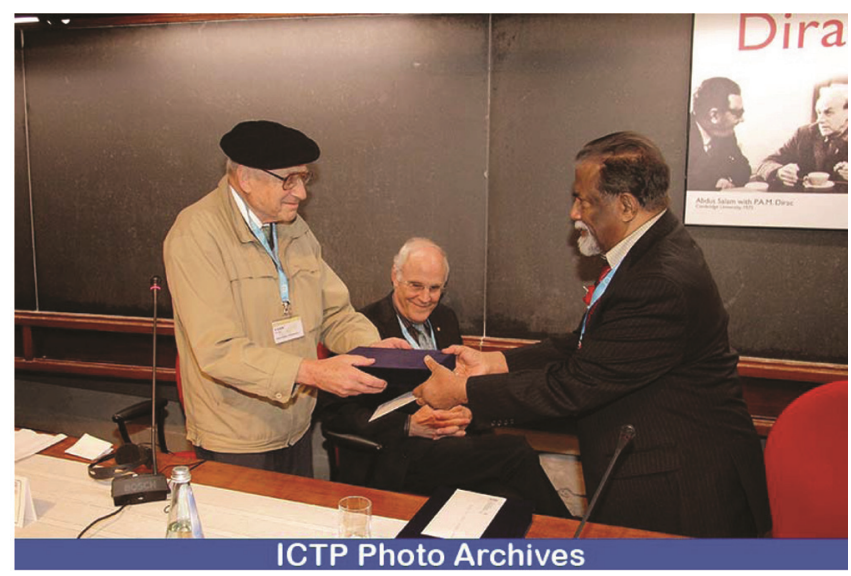

Figure 1. The photograph shows George receiving the Dirac Medal from Walter Kohn (1998 Nobel Prize in Chemistry). Looking on is David Gross (2004 Nobel Prize in Physics). 


\begin{abstract}
The common properties of all weak interactions are striking. In addition to the agreement to within a small factor of all the effective coupling constants, the weak interactions share the common property that parity, isotopic spin, and probably charge conjugation are violated in all of them. The law of conservation of leptons as well as the hypothesis of Universal Fermi Interaction appear as desirable and elegant ordering principles but it is not quite clear as to how far these are obeyed in nature. It seems however clear that the idea of a completely 'symmetric' Universal Interaction and the conservation of leptons cannot together be consistent with all the experiments. However, it is possible to present more or less elegant solutions to the problem, like our centered triangle in which the muon interactions are AV while the others are VT, all with the same coupling constant. The ultimate success or failure of this approach to the theory of weak interactions is to be assessed in terms of direct observational tests. The somewhat unsatisfactory state of the experimental results is rapidly being remedied and one can hope to have deeper insight into the possible common origin of all weak interactions, in the near future and thus into another aspect of the behaviour of the elementary particles of nature.
\end{abstract}

Figure 2. This is the epilogue of Ennackal Chandy George Sudarshan's Ph D thesis submitted in 1957 to the University of Rochester, USA.

speed at zero energy. This interpretation does away with causality objections for the existence of faster-than-light signals (but see Thouless $^{9}$ ) and permits the construction of a consistent theory of tachyons; their stability, however, is another matter. Efforts to detect such particles have been unsuccessful so far. George and colleagues knew that the detection of tachyons would be an improbable event, but wondered if they may have gone unnoticed. The article by Bilaniuk et al. ${ }^{7}$ ends with this endearing question: 'Has any one of you gentlemen discarded a set of data on such account? It may have been caused not by faulty electronics, as you assumed, but by a shower of meta particles.' Experimental readers will especially appreciate this remark (and women readers will likely forgive them). Later, Narlikar and Sudarshan ${ }^{10}$ studied tachyons in the context of cosmology, and concluded that any primordial tachyons that might have been created are unlikely to have survived to the present era.

Though it is well accepted that George and collaborators have the priority on tachyons (the publication itself was delayed because the original version was rejected by Physical Review Letters (PRL) and Bilaniuk rewrote the $P R L$ version in a more understandable style ${ }^{7}$ ), it was not without controversy at first. Bilaniuk et al. ${ }^{7}$ used the name 'meta' particles, and the credit for the fascinating name of tachyons belongs to Gerald Feinberg ${ }^{11}$ of $\mathrm{Co}$ lumbia University, USA. This attractive label and the publicity machinery of Columbia led to the attribution of faster-than-light particles to Feinberg in popular journals (TIME, The New York Times, The New Yorker Magazine). For instance, the Scientific Research ${ }^{12}$ article (signed by a writer identified only the initials H.L.D.) was all about Feinberg's contributions; though it contains phrases such as 'people have argued', 'handful of theorists' and refers to Feinberg himself as 'one of those theorists', it is entirely silent on George or his collaborators. This was upsetting to George, who wrote a tough letter to Feinberg, in which he asked, 'Is it that I am unaware of some- thing that you have contributed to the understanding of faster-than-light particles (other than, of course, the name TACHYONS)?' He concluded with: 'I am baffled as to what you are up to. To me, to say the least, the whole thing stinks.'

George and his co-authors protested to TIME for perpetuating the error. I reproduce in Figure 3 the response provided by TIME some months later; it is mostly selfexplanatory.

There is no record of how Feinberg reacted to George's aggrieved and aggressive note, if he did at all; but in a Scientific American article ${ }^{13}$, he explicitly stated that 'This interpretation of the negative-energy states of the Tachyon was first proposed in 1962 by O. M. P. Bilaniuk, E. C. G. Sudarshan and V. K. Deshpande of the University of Rochester'. The New York Times published Feinberg's obituary ${ }^{14}$, marking his death due to cancer at the age of 58. It makes no claims for tachyons, with much of the original work attributed to him for the existence of two kinds of neutrinos rather than the one that was then postulated. For what it was worth, the credit ultimately belonged to George.

\section{$V$ minus A structure of weak interactions}

I came to know of Sudarshan's V - A work and the priority controversy surrounding it when, sometime during the CTS days, he gave a somewhat sensational interview in Delhi accusing Gell-Mann of unfairness - to put it mildly. That episode stimulated me to find out a bit about the work, and I would like to describe it briefly and comment again about priority issues.

Much has been written about this work and the circumstances surrounding the credit for it, by Sudarshan and Marshak ${ }^{15}$, Marshak ${ }^{16}$ and others at George's 75th birthday meeting ${ }^{5}$ and elsewhere. The companion article by Mukunda ${ }^{17}$ describes it in excellent detail, and delineates the discovery. 
The bread and butter of high-energy particle physics is the Standard Model, which has had huge successes in predicting experimental findings and describes three of the four fundamental forces or interactions (the electromagnetic, weak and strong), and classifies all known elementary particles. The last important confirmation was the discovery at CERN of the Higgs boson in 2012. A critical ingredient of the Standard Model is the electroweak interaction, which is the unification of two of the four forces, namely electromagnetism and the weak into a single electroweak force. Sheldon Glashow, Abdus Salam and Steven Weinberg were awarded the 1979 Nobel Prize in Physics for their electroweak work. Three major steps preceded the foundation of the electroweak theory itself: the local fermion theory of Enrico Fermi, the parity violation theory of T. D. Lee and C. N. Yang (and its experimental verification by $\mathrm{C}$. $\mathrm{S}$. $\mathrm{Wu}$ ), and the last one is the ground breaking universal theory of $\mathrm{V}-\mathrm{A}$ interactions.

$\mathrm{V}-\mathrm{A}$ refers to the vector and axial vector charged currents. Fermi's original theory of weak interactions

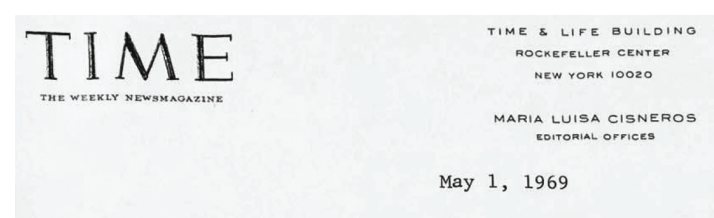

Dear Professor Sudarshan:

It is some time since you wrote about our February 14 story, "Exceeding the Speed of Light," and we very much regret that your letter was not answered before this. It was misfiled and was only discovered today. The letter was, however, considered for publication but because of space limitations in the column, we were unable to include it there. Nevertheless, we are glad to discuss your objection with you now.

In reporting science news in TIME, we are concerned to give proper credit to the person or persons responsible for significant breakthroughs, and we are consequently very sorry about the misunderstanding that has arisen over the abovementioned story $\mathrm{Dr}$. Bilaniuk and $\mathrm{Dr}$. Deshpande have also written about it, as you may know.

Professor Gerald Feinberg was not the first or by any means the only one to work on particles that travel faster than the speed of light, as you point out. But in presenting his argument in terms of quantum physics rather than Einstein's theory of relativity, and in coining the word "tachyons," he theory of relativity, and in coining the word "tachyons," he
has come up with a new concept. The difference between the two equations should have been made clear in our story and credit for research stemming from the Einstein theory should, of course have been given to you, to Dr. Bilaniuk and to Dr. Deshpande. Should we have occasion to report on this particle again in TIME, we will most certainly mention your research and make the distinction between it and Dr. Feinberg's.

Thank you for writing. We were, as we said, glad to hear from you and again express our regret at the delay in replying.

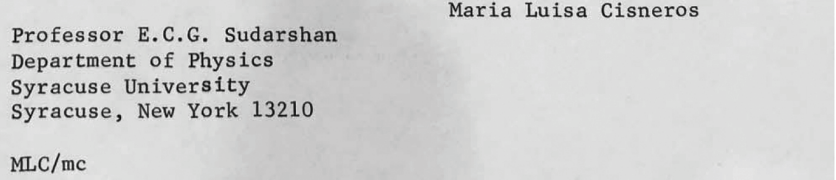

Figure 3. From the editorial office of TIME in response to complaints about unfair attribution. involved only vector (V) currents, but allowed generalization to contain scalar (S), pseudoscalar $(\mathrm{P})$, axial vector (A) or tensor (T) currents. Various combinations including $\mathrm{S}$ and $\mathrm{T}$, as well as linear combinations of vector and axial vector, were used. Several names can be associated with these ad-hoc proposals. The experiments were not all giving the same answer, either. George was the first to propose, led by the concept of chiral invariance, that the interaction is strictly of the V - A type (i.e. parity was violated maximally). As George himself has pointed out, he laid the foundation for the edifice that is now the electroweak theory; obviously, his advisor Robert Marshak played an important role in the development of George's ideas.

The fact that Sudarshan and Marshak were denied the priority for the work and that it came to be known, for some time, as the work of Feynman and Gell-Mann ${ }^{18}$, was the consequence of the poor way in which the publicity and publication of the Sudarshan-Marshak results were handled by the authors, and a certain penchant for publicity on the part of Gell-Mann and Feynman. On the former aspect, many compounding errors took place (see also ref. 17): Marshak did not allow George (for whatever reason) to present the result in the Sixth Rochester Conference (April 1957) that he himself had organized; they put such a key result in conference proceedings whose publication got delayed inordinately; they discussed the results with a highly intelligent competitor without a proper plan to publish their own results quickly, etc. I can only surmise that the combination of an inexperienced graduate student and overly busy professor must have played a role. On the latter aspect, it suffices to quote Lawrence Krauss ${ }^{19}$ : 'Needless to say, word of the Feynman-Gell-Mann paper quickly spread, and poor Sudarshan had to endure talk after talk where he heard the idea for $\mathrm{V}-\mathrm{A}$ attributed to these two leading lights. It was true that Gell-Mann had insisted on an acknowledgment in their paper to discussions with Marshak and Sudarshan, and he always tried to write supportive letters for Sudarshan, and Feynman later acknowledged to Sudarshan that he had since been told that Sudarshan had had the idea for $\mathrm{V}-\mathrm{A}$ before anyone else, and subsequently admitted such in public. But for years, the FeynmanGell-Mann paper became the classic and only reference people quoted when discussing the idea.' There may be a lesson in all of this somewhere for younger scientists.

Marshak has placed on record his correspondence with Feynman and J. R. Oppenheimer, as well as comments on Gell-Mann, and Mukunda ${ }^{17}$ has quoted extracts from them. I can add to this correspondence the stern note that Marshak wrote to Leon Lederman (Nobel Prize in 1998) and the latter's response to Marshak (Figures 4 and 5). This exchange may show the nuances of the wind that was then blowing against Marshak and Sudarshan.

Sudarshan and Marshak ${ }^{15}$ have pointed out that Feynman characterized the situation after a few years as 
'the theory of weak interactions invented by Marshak and Sudarshan, published by Feynman and Gell-Mann, and completed by Cabibbo ...'. It appears that Feynman himself did not know that Sudarshan had the first insight, but the value placed on that work is obvious. For instance, he exulted that 'It was the first time, the only time, in my career that I knew a law of nature that nobody else knew ... It was the only time I ever discovered a new law ${ }^{20}$. Since the paper was authored together by Feynman and Gell-Mann, Feynman's conceding statement may be regarded as shared by Gell-Mann as well. However, the latter has consistently said that, though he learnt about the Sudarshan-Marshak work in a small private meeting organized by Marshak for Gell-Mann (see later), in which George presented his results, he already had the essential idea $^{21}$. In his Caltech Archives ${ }^{22}$, there is a three-page description of the circumstances. It is difficult to distill it in a few words, but I will do so after citing the first half-page of his commentary:

'I began to discuss with him [Arthur H. Rosenfeld] how this [theory of weak interaction] was an attractive idea and maybe experiments were wrong. It made for a universal Fermi interaction, and that was perfectly compatible with an intermediate boson of spin one, which would carry the interaction. It was very appealing, but we were still worried about all these experiments that contradicted this hypothesis.

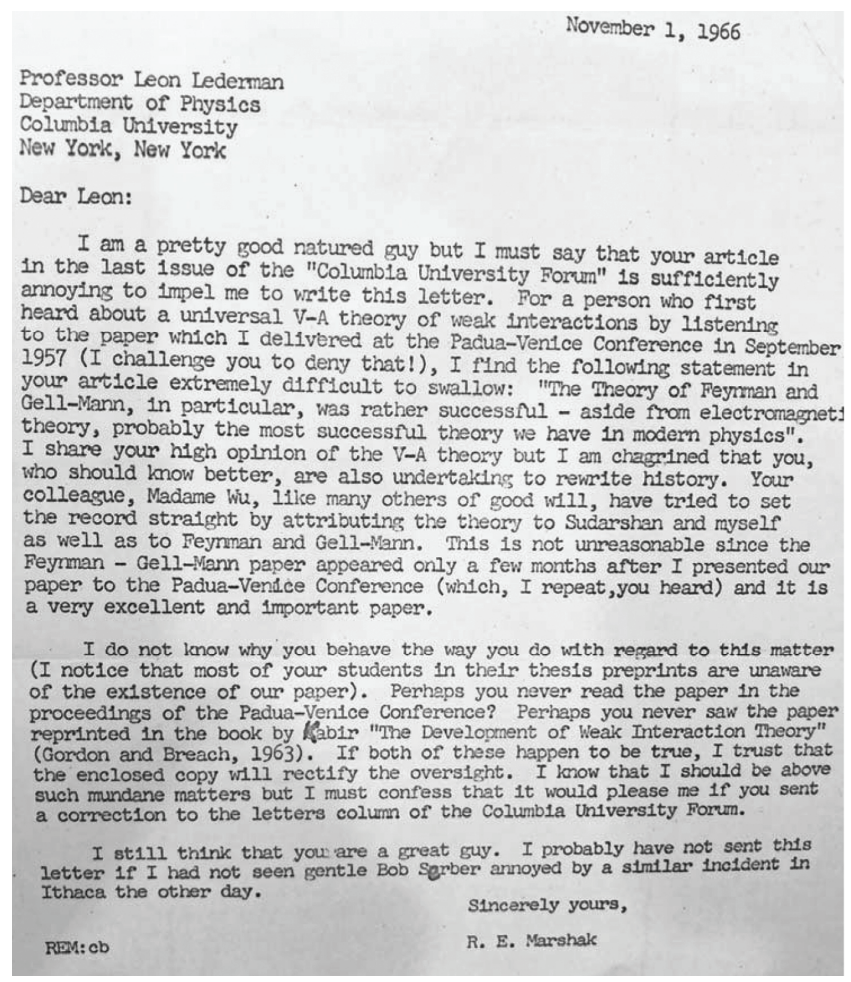

Figure 4. Marshak's note to Lederman stating the priority for the Sudarshan-Marshak work.
'At that time Robert Marshak and his student, George Sudarshan, came to visit. Art and I went to meet with them, and I think with Felix Bloch, at RAND, in Santa Monica. We had lunch together. And they told us about their work, in which they had figured out that these experiments could really be wrong - that they could be criticized and perhaps were actually wrong - lending a lot of strength to this very beautiful, fantastically simple theory of weak interactions. I liked that, and I liked the strengthening of confidence that this might work.

'Art and I had written a section in our Annual Review [of Nuclear Science] called "The Last Stand of the Universal Fermi Interaction," in which we describe how, if all the experiments were really wrong, we could have this beautiful theory. They asked whether we were going to publish any more on it. And I said, "Well, I don't think so. I don't suppose we will. This is what we're going to say, and it says most of it." We always took this very modest approach, and this delaying approach, to publication. I do not know why. [Sighs.]

'So they started writing something also. They started writing on their ideas, which went further, in the sense that they criticized some of these experiments and showed how they might be wrong.'

The interview then states that Gell-Mann went on vacation soon after; that Feynman came back from Brazil,

Columbia University

DEPARTMENT OF PHYSICS

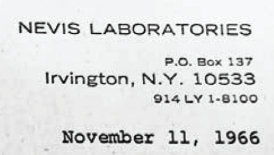

Prof. R. E. Marshak

Physics Department

University of Rochester

Rochester, N. Y.

Dear Bob:

So I've done it again! I did first learn of $\mathrm{V}-\mathrm{A}$ at Venice; I even remember commenting on your courage in defying so many standing results (all of them eventually proved wrong). Althoug significant has suffered the least.

The only mitigation I can cite is that, in my mind, the Feynman - Gell-Mann paper has in it also the CVC theory and, as someone who has been struggling with mu-capture and neutrino major advances of weak interactions. So that although I talked about VA, it is also the extension to high energy weak interactions through CVC (and now PCAC) that was in my mind.

I will indeed communicate appropriately to The Forum.

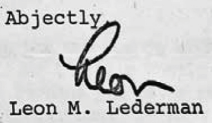

IML: et

Figure 5. Lederman's response to Marshak. 
heard about this theory second-hand and proclaimed that 'now he knows everything', and wrote up his paper from the point of view he had been developing for some time; that Gell-Mann was upset and declared that he, too, would write his own version (it being absurd that Feynman would assume that 'having realized this idea of vector and axial vector he [Gell-Mann] had not realized the rest of it'); that their department chair got the two of them to agree to write one single paper (see earlier extract from Krauss); that Gell-Mann was unhappy with how Feynman wrote major parts of it, but let it go because it was difficult to disentangle how it had been written, etc. At some point, all this needs to be examined by a proper historian. What appears clear is that the idea was startlingly new and it seemed that nobody involved was ready to let go of the issue of priority. Yet, there seems to be a consensus now that George had the broad idea first. I cite a few examples below.

In nominating George for the Dirac Medal, Weinberg wrote this: 'Regarding weak interactions, his greatest contribution was his proposal of the $\mathrm{V}$ minus A theory of weak interactions. The work (done with Marshak, and also later by Feynman and Gell-Mann) was truly a turning point in elementary particle physics. Until the $\mathrm{V}$ minus A theory replaced the previously popular scalartensor theory, it was impossible to interpret the weak interactions in terms of gauge fields. Also the $\mathrm{V}$ minus $\mathrm{A}$ theory led to ideas about conserved vector and partially conserved axial currents, which in turn led to our modern understanding of broken symmetry. I remember how a series of wrong experimental results, culminating in the measurement of recoil moment in helium 6 decay, produced a nearly universal consensus that the beta decay interaction was of the scalar-tensor type. The proposal of $\mathrm{V}$ minus A theory took not only great theoretical insight, but also the courage to go against prevailing opinion. Sudarshan's work was in the best traditions of theoretical particle physics, and deserves to be more fully honoured.' Hegerfeldt expressed the same sentiment when he wrote that '[Sudarshan] was a towering figure in particle physics and quantum field theory. His seminal contributions in this area alone, in particular to the theory of weak interactions and the $\mathrm{V}-\mathrm{A}$ theory, merit any award.'

It is also worth quoting Glashow, who sent the following message for George's 75th birthday meeting ${ }^{5}$ : '[Sudarshan and Marshak] presented a comprehensive analysis of the weak interaction data, which along with the imposition of an elegant symmetry principle, allowed them to deduce a unique form for the weak interactions, the so-called V - A theory. Their daring hypothesis was accompanied by a list of four experimental results that they wrote, 'cannot be reconciled with this hypothesis ... All of these experiments should be redone ... If any of the four experiments stand, it will be necessary to abandon the hypothesis.' This is theoretical physics at its zenith! The experiments were redone with results that now confirmed their hypothesis. It was a stunning accomplishment, yet one which has never been recognized with a prize ... In my view, Sudarshan's seminal contribution to weak-interaction theory, representing only a small portion of his oeuvre, would itself justify the award of a major prize in Physics.'

\section{Sustained links to India}

\section{Intellectual attractions}

George maintained a seamless connection with the Indian scientific community, especially in physics, throughout his life. In particular, he had made in the sixties a few extended visits to Delhi University, Madras University and Matscience. The fact that he had a full-time academic position in the US did not diminish these links. One might say that George's style and spirit did not confine him in geography or in the vast space of ideas. I dwell briefly on this general aspect first before discussing specifics of his involvement in India, even as he kept his long-term centre of gravity in the US.

Since the time George went to UT Austin in 1969, he was directing the Centre for Particle Physics, jointly with Yuval Ne'eman. As a free thinker, he really wanted the Centre to be in a position to appreciate the richness of our universe in broad strokes. In fact, to realize this dream, he hired for something like two years the science historian Jagdish Mehra ${ }^{23}$. George and Mehra organized the 'greatest physics meeting of all time' on the physicist's conception of nature. This expansive idea did not have money to support it in perpetuity. The creation of CTS in IISc was one way of fulfiling that dream; his Directorship of Matscience was another. All this said, I have little doubt that the consistent draw that India had for George transcended the sense of intellectual opportunism: he was in love with India (see Figure 6) and with the notion that he could make a difference to Indian science.

\section{Center for Theoretical Studies}

CTS was in the making for some years before it actually came to life. George had discussed it with D. S. Kothari (then the Chairman of the University Grants Commission) and Dhawan, separately. Sometime in 1971, Kothari and Dhawan appear to have made a joint effort to form the Centre with George as its Director. Other key people involved in CTS at the time were R. Narasimha from aeronautics and K. P. Sinha from physics. CTS came into being in July 1972. Within a few years, others such as N. Mukunda, Madhav Gadgil, Sulochana Gadgil, H. Sharat Chandra, A. K. Rajagopal (who returned to the US within a year), R. Rajaraman, J. Pasupathy and V. Nanjundiah joined CTS. Many of them would most likely not have 
come to IISc without CTS - and without George at its helm.

It was clear from the beginning that George would be present only some three months a year; so the day-to-day operation was to be managed jointly by Mukunda (who came in 1972 from TIFR) and Rajagopal (who came in 1974). When the latter returned to the US a year later, Mukunda carried on the work under the title of 'Professor-in-charge', promptly preparing fortnightly handwritten reports to George about the detailed happenings at CTS. Reading many of those reports with the benefit of hindsight, one aspect that stands out is the easy relationship that existed between Mukunda and George, and the constructive role played by the former.

An undated document announcing the formation of CTS says: "The Center proposes to concern itself with creative research in the theoretical sciences, including elementary particle physics, statistical mechanics, astrophysics, relativity, cosmology, theoretical meteorology and geosciences, chemical physics, solid state theory, applied mathematics, mechanics, systems sciences, mathematical economics, theoretical biology, linguistics, models of social phenomena, theoretical aspects of behavioural sciences, philosophy of knowledge and foundations of science.' George informally declared at the inauguration of CTS that its special feature was to be interlinked theoretical studies-even Malayalam poetry would be fine, he said - not merely theoretical physics. CTS was 'predicated on the thesis that pursuit of excellence and illumination of basic principles is an integral component of the intellectual program of problem solving that we call the scientific way of life'.

In April of 1974, in a National Committee meeting attended by Mukunda, Sinha and others, a suggestion was

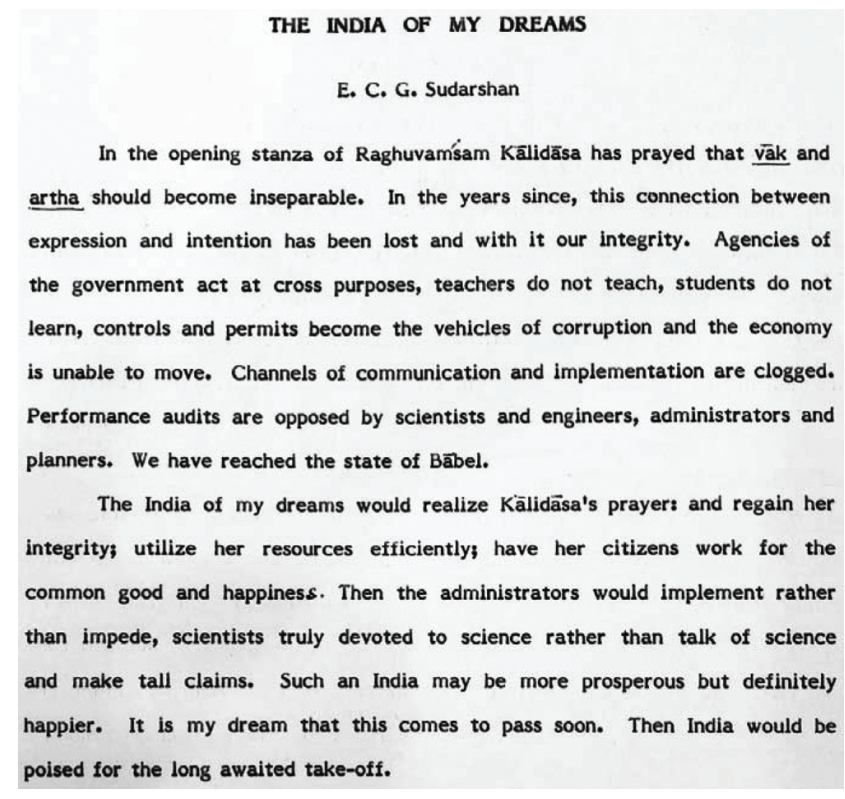

Figure 6. India of George's dreams, probably in the 1970s. made by Kothari to name CTS after the distinguished Indian physicist, S. N. Bose; this suggestion seemed to have had the enthusiastic support of C. Subramaniam, an influential person from Tamil Nadu, a presence in local and national politics for many years, who was also present at the meeting. Mukunda excitedly communicated the gist of this discussion to George, whereupon the latter promptly wrote to Dhawan on 9 May 1974, suggesting this change in name to be taken to the IISc Council if Dhawan favoured it. The matter does not seem to have gone any further, presumably because there was at that time no example in IISc of naming a unit after any name but Tata.

George's admiration of S. N. Bose is obvious from several of his writings. In fact, he had written in December 1972, a letter to Prime Minister Indira Gandhi suggesting an international symposium in Bose's honour, to which she had responded enthusiastically, offering support through the Office of the Minister of Science and Technology. The Symposium did come to pass in July 1974.

CTS was successful in various ways. Part of this success was, of course, George himself, who was instrumental in bringing to IISc much of modern physics. He inspired many young people in research institutions across the country to new areas that were, to a first approximation until then, largely the province of TIFR. George's appeal was not only his high scientific reputation but also the fact that he could explain difficult concepts without jargon and formality, using simple words to lay down their context, revealing their profound nature. And his energy and liveliness came through vividly.

The other part of the success of CTS was the first-rate people it nurtured. Yet, the breadth of CTS's interests and its unconventional administrative status created its own tensions. Over time, various people in CTS were inspired to form other centres at IISc - such as the Centre for Atmospheric and Oceanic Sciences, the Centre for Ecological Sciences, Developmental Biology and Genetics Laboratory, etc. With time, however, CTS lost George's ideal of interdisciplinarity; in fact, at some point, it formally bifurcated into the Centre for High Energy Physics and the Centre for Contemporary Studies. George's connection with CTS loosened well before most of these changes took place.

\section{Institute of Mathematical Sciences}

George had kept up with Indira Gandhi on occasion, whom he regarded very highly ${ }^{24}$. His ambivalence on the National Emergency of 1975 did not change their relationship, and he wrote to her in January of 1976 mentioning the criticism from the West. Mrs Gandhi's response is reproduced in Figure 7. In any case, it appears that George may have told her that he would return to India if 
a suitable position were to open up. He was offered in 1984, the Directorship of the Institute of Mathematical Sciences in Chennai, known better as Matscience then, and he accepted it.

Alladi Ramakrishnan had established Matscience in 1962, with financial support from the Tamil Nadu Government and the political support from C. Subramaniam, ostensibly patterning it after the Institute for Advanced Study in Princeton, USA. It was clear that there was enormous support and excitement within Matscience for George's appointment as Director, and there were many expectations: from salaries and fellowships to buildings to faculty expansion to governance methods, etc. Indeed, during his time, its support base extended to the Department of Atomic Energy, and with Raja Ramanna's support, Matscience expanded significantly in budget and personnel.

Alas, the combination of George's unconventional style, the factitious faculty and a somewhat complex administrative structure led to controversies at Matscience. The fact that Matscience celebrated George's eightieth birthday (Sudarshan Fest, 16 September 2011), and many of the protagonists were present at the event, suggests that most of the irksome details of the controversies have been forgotten, if not exactly forgiven, by both parties. So it is not useful to discuss them, but glossing over them

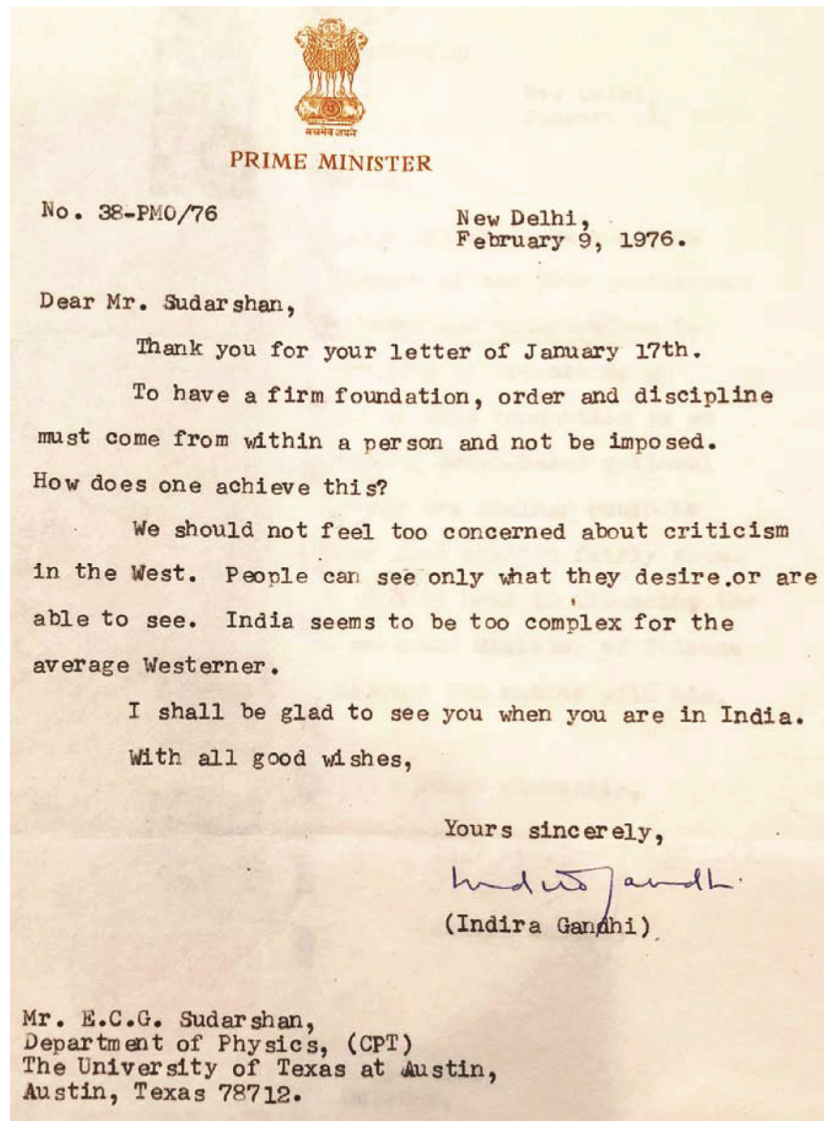

Figure 7. Letter from Prime Minister Indira Gandhi during the period of National Emergency. without any remarks makes little sense, especially because it had an impact on George. He believed that his side of the story did not get enough exposure.

One of the issues that has been stated to me in various conversations is that George's eminence could go only so far to compensate for his prolonged absence from the country; and that, even when he was in the country, he was not always at Matscience. As a speaker at various meetings, George was in high demand, which he met meticulously at some cost to himself and his institution. There is no question that he worked very hard but some of his decisions were countermanded, resulting in impatience on his part. Changes in long-standing traditions, even bad ones, can happen only slowly, requiring constant nudging from the top; nothing happens by fiat, especially in India where non-cooperation has been honed to perfection. George's American-style accountability had to do with results, not with how you got there.

\section{George's world-view}

\section{Self-made philosopher}

Very few men have the luxury of choosing their names, but George did. He chose 'Sudarshan' for his last name (he was previously known as E. C. George), and regarded himself as a Vedantin. Even as a young person, he had read some Upanishads and Hindu mythology from his father's library, and was attuned to Upanishadic thinking so the conversion from Christianity was naturally suited to his temperament. Part of the motivation was his marriage into a well-known and accomplished Brahmin family in Bangalore. He was quite well versed in ancient texts and could recite various classical Sanskrit prayers. His numerous writings show that he was in love with the Upanishadic imagination; in particular, the rationalism of the Advaita philosophy suited his taste for asking deep questions of unification in modern particle theory. He seemed to have found harmony in his physics as much as in his philosophy, and was constantly trying to integrate them both. At one time he expressed the view that C. P. Snow's talk of two cultures perhaps merely expressed one culture in two different ways.

While George loved Yogic philosophy, he was not always an embodiment of equanimity that it extolls. At his best, however, he saw unity in human thought as a whole; and he made no distinction between India and the US in how he conducted himself and how much he gave each country.

One other comment is useful to add: in spite of his willingness to talk to anyone that sought him out, George was an elitist in the following sense. While he was willing to pay homage to the 'collective wisdom' of scientists, acquired through pooled resources, he thought that each worthy scientist ultimately makes a choice: who are 
his fellow scientists whose words he will accept on what is worth exploring.

\section{Between the two worlds}

George lived for about $70 \%$ of his life in the US but always thought of himself as a bridge between two cultures, Indian and Western. He thought that, as an Indian who lived in the West, he had an especially heightened appreciation for the marvels and shortcomings of both countries and cultures. It was somehow through the synthesis of the two worlds that one would experience the whole - and George thought that he might have been in a good position to accomplish that.

But one can also see that he appreciated the limitations of this potential. For instance, George also felt that he did not have a devoted constituency anywhere. 'If you do not have a constituency,' he said, 'you should be able to transcend disappointments'. He felt that making compromises meant that one was repudiating one's culture and seeking patronage; he stated that one has to be prepared to be lonely if one dares to be different.

His love for India notwithstanding (and he remained an Indian citizen until he was about 60), George would not live permanently in India because he thought that living in the US more resembled the old forest dwelling in Puranic times - in so far as its modern conveniences freed up more time for contemplation. He once said that, while he could easily merge in an Indian crowd, he had lost most of the survival skills, especially in the academic world (which he regarded as the most political, everywhere). He certainly felt wronged on more than one occasion by the scientific community at large, and that 'international science was not a monarchy nor democracy; rather it is reminiscent of a more primitive social organization of the era of robber barons'.

\section{Science and spirituality}

For many scientists, spirituality is an unnecessary distraction. But George saw a spiritual dimension in his scientific work and wore it on his sleeves, as it were, just as he wore his Indian-ness. Disciplined observation, experimentation and analysis of the external world are the demands of a serious scientific pursuit; he felt that this same discipline could be directed inwards as well. The important element in this process is not any particular scientific fact or a preferred branch of science; rather, it is the scientific path itself. Both paths involve uncharted territories, both involve personal dedication and discipline, and both are creative and joyous. For George, ideas such as 'divine grace' were not abstract concepts; he equated this particular idea with the inspiration that a scientist derives on rare occasions; he saw it as a causeless illumination that can come only from beyond oneself.
In short, he did not see science and spirituality as distinct operations needing unification; they were already united.

The views I have attributed to George are sprinkled through many of his public talks and private letters. The breadth of his shifting interests, and his willingness to openly explore them in public talks - of which there were many - encouraged some people to believe that he was 'somewhat strange'; this reputation did not work to his advantage.

As is well known, by the late seventies, George got actively interested in Maharshi Mahesh Yogi, TM, the relationship between theoretical physics and consciousness, etc. I guess that he always thought that these matters, including such effects as levitation ${ }^{25}$, were within the realm of physics, and therefore quite legitimate areas to study. He believed that he could expand the scope of physics in unique ways, while a few of his science friends thought that he was going astray and were disappointed; it irritated them further when George responded with what appears as somewhat of an arrogance that he might be the one, chosen or otherwise, to uncover the deep connection between mysticism and physics. To these same people, however, his association with the philosopher Jiddu Krishnamurti, lasting more than a decade, might have seemed less objectionable. In truth, George was following no fads but his inner dictate.

\section{Awards and honours}

Sometimes awards and honours make a scientist's reputation. How many Nobel laureates would have remained obscure without that recognition! George was anything but that: many people in different continents admired him for his physics and for the pleasure he derived from it, and his name meant something to vast communities of people. He received several awards, including the Dirac Medal of the ICTP, the TWAS Prize and the Majorana Prize. In India, he was awarded the C. V. Raman Prize, the S. N. Bose Medal, and the Padma Bhushan as well as the Padma Vibhushan, among others. But they do not define the man.

It was clear to George that his $\mathrm{V}-\mathrm{A}$ work, which he thought was his best, deserved greater formal recognition than it received. It is not clear when exactly he thought that it was of Nobel caliber; he was certainly nominated multiple times, the last one probably in 2001, but was not chosen for whatever reason. It would be certainly tiresome to know that one was nominated multiple times (as he did), and to discover each time on the appointed day that it did not happen. He does not seem to have thought that his quantum optics work would be awarded the Nobel Prize, but was inordinately irritated when it was given to Roy Glauber for work that he (and many others) regarded was his. The fact that he had been twice passed over for this coveted honour certainly bugged him 
but it did not stop his creativity. Perhaps his admirers made it harder for him to forget that an injustice was served. To have to fight for credit for the V-A work, Tachyons, Diagonal Representation, perhaps others; to have to face a disappointment with his Matscience leadership, the fact that he was not made a distinguished professor at Austin (despite other honourific treatments); that his friends did not speak up when it mattered most, and so forth, must have weighed on him at times. On top of these skirmishes, the disbelief that his mixing of spirituality and science caused in his friends, the first marriage that ended badly, his fight with long-standing diabetes, etc. took a toll as well. Despite these unpleasant events in his life, George travelled extensively, spoke at many meetings with great energy, developed friends in several continents, took deep pleasure in his beloved physics and was amazing to the end. This is the George Sudarshan that we should best remember.

Since George was fond of Indian mythology, I may be forgiven for thinking of him as Vishwamitra-like. As is well known, the great sage Vishwamitra gained many merits along the way to attaining the Brahmarshi status that was denied to him multiple times by the establishment. He created out of his sheer power of tapas an entire universe for Trishanku. Alas, Vishwamitra, too, occasionally faltered because of his imperfections.

\section{References and comments}

1. The shloka reads: vaasanaad.vaasudevasya vaasitam te jagat.trayam sarva.bhuuta.nivaaso.si vaasudeva namo.stu te. It can be found towards the end of Vishnu Sahasranama, which itself is part of Anushasana Parva - literally, the 'Book of Instructions' - in the Mahabharatha. Its meaning is: Vasudeva's fragrance permeates all three worlds, And all life resides in Him: Vasudeva, I bow to you. Its relevance to the title of the talk, which mystified me then, and does so even now, can perhaps be understood only by appreciating Sudarshan's lofty sense of universal being.

2. At that time I knew only two works of Raja Rao. Kanthapura, written in 1938, was a prescribed text for college students at one time, and I had been amused and impressed by how the author described common native rituals naturally without being stifled by English as the medium. In The Serpent and the Rope, published in 1960 , the author discussed in a charmingly autobiographical style, weaving between France and India, such concepts as Existence, Illusion and Reality. The title is a reference to the central concept of the Advaita, of ignorance that leads to the mistaking of Illusion for Reality, the rope for the serpent.

3. The citation for the latter reads: 'for his lifetime achievements in different branches of theoretical physics; especially for being the first to propose the Universal V-A Theory of the weak interactions, and to formulate the Quantum Optical Equivalence Theorem, the predictions of both of which have been confirmed by experiments in their minutest details'.

4. Rothman, T. and Sudarshan, G., Doubt and Certainty, Perseus Books, 1998. The book is not perfect by any means and has many ideas patched together, somewhat glibly on occasion, but the sheer scope and breadth of topics covered is admirable, often entertaining: quantum mechanics, particle physics, cosmology, symmetry, chirality, Zeno effect, metaphysics - everything that seems to be needed to be appreciative of Nature's ways. Rothman was a tem- porary faculty member in the Applied Physics Department in my School, so I got to know how the book came about. By the way, Sudarshan did not take any royalty from the book.

5. The papers presented at the 75 th birthday meeting in Austin, 'Sudarshan: Seven Science Quests', appeared in full or as abstracts, in J. Phys: Conf. Series, 2009, 196. In his Dirac Medal lecture, Sudarshan said that the number 'seven' was chosen for reasons of symmetry with his age.

6. The selection committee did not advance Sudarshan in the first year of the nomination, but chose him next year. I was somewhat disappointed because I left my position at ICTP a few months prior to the award ceremony. I went back to ICTP to be part of the ceremony, but had to return to New York; as irony would have it, a few hours before the lecture; thus, I have had to content myself by watching it on YouTube. All I could do, even though I was no longer in charge, was to assign a person to take care of Sudarshan's logistical needs; he was not particularly well by then. The Dirac Medal was shared with N. Cabibbo (posthumous). The citation for Sudarshan reads: 'Sudarshan's important contributions to theoretical physics include the discovery (with Robert Marshak) of the V-A theory of weak interactions, which opened the way to the full description of the unified electroweak theory. He has also made innovative discoveries in the field of Quantum Optics, including the Optical Equivalence Theorem, which provides the foundation upon which the investigations of the manifestly quantum or non-classical character of the electromagnetic field are based'. Towards the end of his Dirac lecture, Sudarshan says something as follows (not verbatim but close): 'My friends tell me that my work is not fully appreciated because I publish them in different journals as short papers ... and I was pleased that the Proceedings of the Conference for my 75 th birthday highlighted my contributions; the contributors were all my friends who would not steal anything from me.' Somehow, that statement seemed to be Sudarshan's own assessment of his scientific interactions; he seemed somewhat at a loss and a shadow of his earlier self.

7. Bilaniuk, O. M. P., Deshpande, V. K. and Sudarshan, E. C. G., 'Meta' relativity. Am. J. Phys., 1962, 30, 718.

8. Bilaniuk, O. M. P. and Sudarshan, E. C. G., Particles beyond the light barrier. Phys. Today, 1969, 22, 43.

9. Thouless, D. J., Causality and tachyons. Nature, 1969, 224, 506.

10. Narlikar, J. V. and Sudarshan, E. C. G., Tachyons and cosmology. Mon. Not. R. Astron. Soc., 1976, 175, 105.

11. Feinberg, G., Possibility of faster-than-light particles. Phys. Rev., 1967, 159, 1089.

12. Are there particles faster than light? Sci. Res., August 1967, p. 34.

13. Feinberg, G., Particles that go faster than light. Sci. Am., 1970, 222, 68 .

14. The New York Times, 23 April 1992, obituary section, p. 24: 'Gerald Feinberg, 58, Physicist; Taught at Columbia University'.

15. Sudarshan, E. C. G. and Marshak, R. E., Origin of the universal V - A theory. AIP Conf. Proc., 1994, 300, 110. In this paper, the authors post their opinion of the Feynman-Gell-Mann paper as follows: 'Apart from the priority question - which seems easy to resolve - it is difficult to see how the mass reversal invariance argument improves upon chirality invariance in 'deriving' the universal V-A interaction'. As far as Sudarshan was concerned, chirality invariance was the most fundamental level of understanding possible; he seemed to have thought that everything else was a bit of obfuscation.

16. R. Marshak discussed this a few times. The gist can be found in the Oral History Interview of Marshak, posted on the AIP website, towards the end of Session IV. It also describes how J. J. Sakurai's paper got written on the same subject (Nuovo Cimento., 1958, 7, 649) - with some crucial input from Marshak, but no acknowledgement of this by the author. Marshak's recollection of events on the weak interaction work is also described in his after-dinner 
lecture at the 60th birthday celebrations of George Sudarshan, 'The pain and the joy of a major scientific discovery', 1991. He regretted that he did not do enough to promote Sudarshan's work.

17. Mukunda, N., The life and work of E. C. George Sudarshan. Curr. Sci., 2019, 116(2), 000-000.

18. Feynman, R. P. and Gell-Mann, M., Theory of Fermi interaction. Phys. Rev., 1958, 109, 193.

19. Krauss, L. H., Hiding in the mirror. Resonance, 2011, xx, 801.

20. Feynman, R. P., Surely you're joking, Mr. Feynman!, Norton \& Co, New York, USA, 1985, pp. 250-251.

21. Around 2005, I had a relatively long conversation with Gell-Mann about this topic (and others), during a ride from Los Alamos to Albuquerque when we were the only two passengers in a limo. My recollection is that he made somewhat different statements during our meeting from the published version referenced below, but I will stick with the latter.

22. M. Gell-Mann's interview with S. Lippincott, Caltech Archives, 1997.

23. Jagdish Mehra is the author of Richard Feynman's biography. The Beat of a Different Drum: The Life and Science of Richard Feynman, 1994 and several volumes on The Historical Development of Quantum Theory.

24. On 6 March 1984, Sudarshan nominated Mrs Indira Gandhi for the Nobel Peace Prize as the 'voice of peace and social justice throughout the world'. Alas, she was assassinated in October of that year.

25. In his presidential address of the Psychical Society-see Proc. Soc. Psychical Res., 1919, 30, 273-290 - Lord Rayleigh, an out- standing scientist, honoured with the Nobel Prize for discovering argon, the Cavendish Professor to succeed J. C. Maxwell, President of the Royal Society, etc. had this to say: 'Scientific men should not rush to conclusions, but keep their minds open for such time as may be necessary... Our goal is the truth, whatever it may turn out to be, and our efforts to attain it should have the sympathy of all, and I would add especially of scientific men'. He also added: 'Every tiro now knows that the stones to be seen in most museums had an origin thought impossible by some of the leading and most instructed men of about a century ago'. The concern is whether this benevolent and obviously judicious principle allows a lot of crackpot ideas to flourish; and the real question is whether the observations in question can be repeated in many environments and are subject to strict experimental control.

ACKNOWLEDGEMENTS. I thank Professor Rohini Godbole and her editorial team for inviting me to write for this collection. Professor S. M. Mahajan shared his unique perspective on various fronts concerning George Sudarshan and Professor N. Mukunda answered several of my questions patiently. He and Professor R. Narasimha shared their memories of the origins of CTS. I reiterate my debt of gratitude to Professor G. Bhamathi. The draft of this essay was improved because of the comments from Professors C. S. Aravinda, G. Bhamathi, R. Godbole, S. M. Hanasoge, S. M. Mahajan, N. Mukunda, R. Narasimha and R. Roy but they cannot be held responsible for its lapses.

doi: $10.18520 / \mathrm{cs} / \mathrm{v} 116 / \mathrm{i} 2 / 216-226$ 